\title{
ENSAYOS
}




\section{¿CONTRIBUYE LA INVESTIGACION SOCIAL A LA TOMA DE DECISIONES?}

José Joaquín Brunner*

Me adelanto a decir que mi exposición tiene menos el carácter, siempre un poco solemne, de una "conferencia inaugural" y está más en la naturaleza de una reflexión entre amigos y colegas. En efecto, el tema anunciado - “investigación educacional y toma de decisiones" - se presta demasiado fácilmente para una cierta retórica tradicional de lamentos, cuando en verdad lo que necesitamos hacer es explorar los cambios que están ocurriendo a nuestro alrededor en términos del binomio investigación social y decisiones.

Tratándose, como se trata aquí, de una reflexión bastante tentativa, me tomaré la libertad de abordar varios temas que, a pesar de su aparente dislocación, me parece están íntimamente conectados. Primero, esbozaré la relación entre conocimientos y su utilización práctica considerada desde el punto de vista de la imagen social de los intelectuales. Luego abordaré la cuestión de la utilización de conocimientos vista a través de dos modelos de concebir su incorporación a los procesos de decisión. En seguida formularé algunas apreciaciones, a partir de estudios y de mi experiencia, sobre la participación de los investigadores educacionales en los procesos de formulación y decisión de políticas. Por último, desarrollaré varios argumentos destinados a explorar lo que me parece es el nuevo contexto en que se desenvuelven las prácticas de conocimiento que a nosotros nos interesan.

Para partir daré una brevísima mirada a la figura del intelectual, de cuya imagen pública los investigadores sociales somos algo así como herederos empobrecidos. En un reciente libro sobre la modernidad, Leszek Kolakowski (1990: 63) ha escrito esta frase lapidaria:

"En las almas de los intelectuales se da una lucha sin fin. Están desgarrados entre el sentimiento de su superioridad, su misión especial, y la secreta envidia hacia hombres cuyo trabajo tiene efectos visibles y verificables".

En ninguna parte ese desgarro es más visible que allí donde se entrecruzan la política y la vida intelectual; el poder de mandar — con sus oficinas, rangos y símbolos de prestigio- y el poder de crear -a través de la investigación, la reflexión y la comunicación- conocimientos y medios simbólicos de acción.

De esa contraposición surgen dos imágenes por completo distintas del intelectual. A un lado están aquéllas que representan al intelectual como un resentido. Un personaje usualmente investido de una percepción altruista de sí mismo, que pontifica a los demás sobre cómo conducir los problemas públicos, pero cuyas credenciales son al fondo dudosas y que frecuentemente vive exasperado por la escasa valoración -material y simbólica- de su actividad. Paul Johnson (1988) no hizo más que popularizar esta visión, cuyos antecedentes se encuentran en toda la corriente anti-intelectualista que acompaña al desarrollo de la cultura capitalista.

\footnotetext{
* Conferencia pronunciada en el seminario La Investigación Educacional Latinoamericana de cara al año 2000. Consejo Latinoamericano de Ciencias Sociales Comisión Educación y Sociedad, Punta de Tralca, 4-6 de junio de 1993.
} 
Al lado opuesto encontramos las imágenes afirmativas del intelectual. En general, lo representan como el articulador de una función necesaria en la moderna división del trabajo. Son vistos, entonces, como constructores de consensos, portadores de innovaciones o nuevas soluciones, identificadores de problemas, mediadores simbólicos o como quienes aportan la crítica que toda sociedad necesita para no anquilosarse.

No resulta difícil identificar ambas visiones con posturas, respectivamente, "conservadoras" y "progresistas" de la historia, lo que en ninguna parte es más claro que en esa zona donde se entrecruzan la política y el ejercicio de las palabras que moldean las concepciones del mundo. Para una sensibilidad conservadora, en efecto, el intelectual que transita por el campo de las decisiones políticas usualmente revela su completa inhabilidad para los negocios prácticos y fácilmente se convierte en un sofista y retórico. Su presencia estorba. Cuando pretende transformarse en conductor y gobernante, sus sueños personales de ordinario terminan en pesadilla para los demás.

En cambio, una sensibilidad progresista habitualmente valorará la participación de los intelectuales en el campo político. Para eso reclama su compromiso y, si puede, lo instrumentaliza. Exalta el papel articulador y racionalizador de intereses dispersos que cumple el intelectual, su capacidad de expresar demandas sociales y de aportar su ciencia a los procesos de toma de decisiones.

Dejemos hasta aquí este esbozo.

En efecto, deseo revisar ahora esa disyunción de visiones $-\mathrm{y}$ ese desgarro del intelectual- en un terreno más acotado y complejo: el de los investigadores sociales. También aquí coexisten dos matrices básicas —o paradigmas- que postulan entender, y prescriben cómo operar, la relación entre conocimientos y procesos de decisión pública (Lindblom, 1990).

En un lado ubico a las posiciones que haciendo un verdadero acto de fe en las ciencias desembocan en una actitud favorable a la ingeniería política y social. En el otro, a aquéllas que se acercan a lo que, a falta de mejor término, puede llamarse un concepto de autorregulación de la sociedad, el cual supone una concepción diversificada del conocimiento, una valoración de las prácticas locales que llevan a adoptar decisiones y conduce, por lo mismo, a una actitud escéptica frente a las pretensiones de la ingeniería política y social.

Al lado del modelo iluminista o ingenieril, las ciencias, incluida las ciencias sociales, juegan un papel central. Proporcionan las bases para el desarrollo material de las sociedades y los conocimientos e instrumentos para mejorar la vida social, organizar el gobierno de los asuntos públicos y resolver los problemas de control simbólico de la población. El énfasis está puesto aquí en la necesidad de racionalizar los procesos de decisión y coordinación, mediante la incorporación de componentes de información y conocimiento producidos por la investigación social. Por lo dicho, este modelo favorece a la política -y las políticas- como medio para coordinar sistemas complejos. Sólo por esa vía podrían elevarse los niveles de racionalidad estratégico-instrumental del conjunto, resolver eficientemente los problemas de mal funcionamiento que pudieran presentarse y dar una conducción de horizonte largo a dichos sistemas. Consecuentemente, este modelo valora también el papel que desempeñan los órganos decisores y ejecutores del 
Estado, trátese de ministerios, agencias públicas u organizaciones representativas del cuerpo político.

En cambio, el segundo modelo, el que profesa la autorregulación, pone su confianza en procesos de decisión y coordinación que nacen de contextos interactivos donde participan diversos agentes dotados de información parcial y conocimientos locales. Aquí los resultados de la investigación social están llamados a desempeñar una función limitada, al lado de múltiples otros tipos de conocimientos. Lo que se busca no es racionalizar los procesos decisorios sino permitir que los agentes participantes estén en condiciones de indagar por su propia cuenta en un proceso abierto que lleva a mutuos ajustes y arreglos y a producir cambios no previstos; ni siquiera, muchas veces, buscados. Por lo mismo, este modelo favorece la generación de contextos relativamente autónomos de interacción como medio para coordinar sistemas complejos. Por esa vía se espera elevar los niveles de productividad y adaptación de los sistemas, resolver problemas a la manera del "muddling through" —o sea a través de procesos "embarrados" o "con embarradas" - y dar una autoconducción negociada a dichos sistemas. Consecuentemente, este modelo valora las capacidades de autoaprendizaje de los agentes y organizaciones y trabaja con el supuesto de soluciones parciales e incompletas, de ensayo-error, concibiendo a la política $-\mathrm{y}$ las políticas- como una esfera de menor gravitación.

Miradas las cosas desde un punto de vista microsociológico, la relación entre investigación social y toma de decisiones postulada por el primer modelo describe una trayectoria convergente. En algún punto de esa trayectoria, en efecto, se espera que los conocimientos lleguen a fundar decisiones 0 , a lo menos, a iluminarlas, informarlas o respaldarlas. Esto puede ocurrir de varias maneras. La más usual es aquélla postulada por la escuela del "problemsolving", que constituye una especie de versión refinada y acotada de la ingeniería social. Según esta visión, sería posible una aplicación directa de los resultados de una investigación específica a una decisión pendiente. La expectativa es que la investigación proporcione evidencia empírica y conclusiones que sirvan para resolver un problema. El tipo de conocimientos utilizables en los procesos de decisión es variado, abarcando aspectos cualitativos y de proceso, descripciones cuantitativas, construcción de indicadores, relaciones estadísticas o más generales entre factores, etc. (Lindbbom y Cohen, 1979). En cualquier caso, se postula que evidencias empíricamente fundadas pueden llenar un vacío de información o conocimiento, clarificar una situación a la mano y reducir consiguientemente la incertidumbre en que debe tomarse una decisión. La investigación requerida puede pre-existir al problema y ser seleccionada en base a esa necesidad o puede ser directamente comisionada durante el transcurso del proceso de decisión, caso este último en que se estima que el conocimiento producido podría ser utilizado más fácilmente, con mayor impacto y direccionalidad.

Según los estudios disponibles, las expectativas generadas por esta visión sobrepasan con mucho su efectividad empíricamente constatada. Sólo ocasionalmente ciertas investigaciones parecen tener incidencia directa sobre decisiones pendientes; habitualmente, en el caso de decisiones de nivel menor frente a problemas nítidamente delimitados. En efecto, para que ocurra esa aplicación directa de conocimientos a decisiones pendientes se requiere un conjunto extraordinario y concatenado de circunstancias que difícilmente concurren en la práctica. Según señala un estudio, se requeriría "una situación de decisión bien definida, un conjunto de actores de políticas que tengan responsabilidad y jurisdicción para hacer la decisión, un problema o asunto cuya resolución dependa en cierta medida al menos de mayor información, identificación de la necesidad de información, investigación que provea esa información en términos que 
calcen con las circunstancias dentro de las cuales la decisión será hecha, resultados de investigación que sean definidos, no-ambiguos, sólidamente fundados y poderosos, que lleguen oportunamente a los decisores que trabajan sobre el problema en cuestión, que sean comprensibles y comprendidos, y que no entren en conflicto con intereses políticos fuertes". (Weiss, 1979: 34).

En el caso del segundo modelo, en vez de suponerse una trayectoria de convergencia entre investigación y toma de decisiones, se parte del supuesto que la acción social genera una gran variedad de "arenas de decisión". En ellas participan múltiples actores, todos ellos dotados de conocimiento local, información parcial y un capital acumulado de prácticas. Al ponerlos en juego interactivamente buscan arribar a la "solución" del problema, que puede consistir nada más que en su desplazamiento, transformación o simplemente en "pasar" a través de él conforme los actores se las vayan arreglando ("muddling through"). También el conocimiento producido por la investigación se incorpora a esas "arenas de decisión", entrando en competencia o imbricándose con el conocimiento local provisto por los agentes participantes. Por tanto, no estamos aquí frente a una trayectoria de convergencia sino a una serie de procesos que abarcan un conjunto relativamente desordenado de interacciones y de "idas y venidas" de la información y los conocimientos, los cuales eventualmente pueden contribuir, o no, a tomar una decisión. Dicho en otras palabras, la utilización de resultados de la investigación social —cuando se produce- ocurre en "arenas" que se hallan saturadas de conocimiento, el cual se mueve impulsado por las diversas estrategias que los agentes ponen en acción.

En suma, desde el punto de vista de este segundo modelo, los agentes —incluyendo a los funcionarios decisores- producen interactivamente ciertos arreglos más o menos inestables, para lo cual utilizan información y conocimientos tamizados por consideraciones instrumentales o estratégicas. Además, operan siempre en contextos donde la comunicación está sistemáticamente distorsionada por la asimetría en la distribución de recursos de influencia y control. Es bajo esas condiciones, por tanto, que los conocimientos producidos por la investigación social podrían llegar a incidir, limitadamente, en los procesos de toma de decisiones y "solución" de problemas.

\section{III}

Me gustaría ahora cambiar de registro y explorar algunas implicaciones de lo que llevo dicho, empleando como ilustración la investigación educativa y trayendo a colación mi propia experiencia como participante marginal en esa zona donde se entrecruzan el campo académico y ciertas "arenas de decisión".

Me limitaré a hacer tres observaciones.

\section{Algo más sobre la "conciencia desgarrada" y sus expresiones}

La afirmación hasta aquí implícita —de que no existe la pretendida convergencia entre investigación social y procesos de decisión- pareciera oponerse a la percepción que los propios investigadores tienen de su trabajo y del uso que reciben los resultados de su investigación. 
En un estudio reciente de Guillermo Briones (1990), referido a Chile y que cubre el período 1980-1989, la gran mayoría de los 41 investigadores educacionales entrevistados —un $90 \%$, para ser preciso- contestó positivamente a la pregunta sobre si "algunos resultados de sus investigaciones han tenido alguna forma de utilización, cualquiera sea su modo o nivel". Al mismo tiempo, sin embargo, un $80 \%$ de ellos estima que en Chile, en general, el nivel de utilización de los resultados de la investigación educacional es "bajo", mientras los demás sostienen que es apenas "regular". Esta perceptible disonancia puede deberse a que, usualmente, somos más realistas -incluso nosotros los investigadoresa la hora de analizar fenómenos ajenos que cuando somos llamados a dar cuenta de las expectativas que nos hacemos sobre la relevancia de nuestro propio trabajo.

Según los investigadores encuestados, el principal usuario de resultados sería el Ministerio de Educación, aunque sólo un 15\% declara que sus investigaciones fueron destinadas a las autoridades del sistema escolar. Al mismo tiempo, más del $90 \%$ de los entrevistados consideró que "muchas personas que adoptan decisiones en educación no toman en cuenta los resultados de la investigación cuando son incompatibles con sus creencias o posiciones políticas"; un $87 \%$ estimó que las decisiones en educación se basan mas en conveniencias políticas que en fundamentos que pueda proporcionar la investigación educativa, y dos terceras partes se pronunció de acuerdo con la afirmación de que "la mayoría de los investigadores desconoce las necesidades de información que tienen las autoridades educativas para la formulación de políticas y la toma de decisiones".

Sobre la base de una reducida muestra de altos decisores en el ámbito del Ministerio de Educación, el mismo estudio arriba a la conclusión de que el uso efectivo de resultados de investigación, en ese ámbito, es en extremo modesto. Los decisores justifican ese bajo nivel de utilización con argumentos que son conocidos:

- debido a la hora de usarlos, los resultados no están disponibles

- debido al recargo de acciones de administración de corto plazo y la falta de equipos estables de asesoría que procesen la información

- debido a los constantes cambios en la investigación y su tendencia a "autoalimentarse" de investigaciones similares

- debido a la ausencia de investigaciones pertinente

- debido a la desconfianza que provocan investigaciones cuyas premisas se expresan como dogmas, etc.

En suma, después de constatar que los decisores no tenían conocimiento prácticamente de ninguna de las investigaciones publicadas a lo largo del período 19801989, Briones concluye que, "no hay utilización de los conocimientos generados por esas investigaciones en la formulación de políticas y toma de decisiones a nivel superior". Frente a tal diagnóstico, en apariencia desolador, caben varias actitudes.

\section{Respuestas frente a la no convergencia entre investigación y políticas}

Suele decirse que la escasa o nula utilización de resultados de investigación detectada por Briones, y confirmada por otra decena de estudios similares, podría 
resolverse mediante ajustes técnicos en uno, dos o los tres vértices del sistema triangular de utilización de conocimientos; esto es, mediante ajustes en el modo de producción de conocimientos, en su difusión o comunicación, o en el polo de recepción o utilización.

Por ejemplo, algunas fundaciones y agencias internacionales han insistido, no sin éxito dado que son importantes proveedores de recursos, que en las disciplinas sociales la investigación debería orientarse más nítidamente hacia las políticas públicas; o sea, transformarse en investigación "policy oriented". En otros casos esos mismos organismos, al igual que los gobiernos, han enfatizado la necesidad de "focalizar" la investigación, dirigiéndola hacia grupos-objetivos seleccionados entre los sectores más necesitados de la población. Además, los organismos financiadores tienden a incluir, entre los criterios de elección de proyectos y asignación de recursos, consideraciones de "pertinencia" o de "impacto" previsible de la investigación, todo esto con el objeto de incidir sobre la producción de conocimientos.

No me detendré a analizar cómo cada uno de esos estímulos genera una adaptación táctica de nosotros los investigadores, forzados por las circunstancias a acompañar un juego que, muchas veces, nos merece reparos 0 , en cualquier caso, nos provoca fundadas dudas. Sólo quiero decir que, en el fondo, cada una de esas medidas destinadas a aumentar la "relevancia" de la investigación para las políticas supone el modelo ingenieril o de convergencia, y descansa sobre la premisa implícita de que el conocimiento puede ser aplicado directamente a la solución de problemas mediante un efectivo diseño y orientación de su producción.

Algo similar ocurre con las medidas que se proponen para mejorar la difusión o diseminación de resultados de investigación. En efecto, suele afirmarse, como se establece en un documento experto del año 1987, que "el problema radica en que existiendo abundante información acumulada en América Latina sobre educación, ésta no se utiliza plenamente en la toma de decisiones" (Corbalán, 1987). Sobre la base de ese diagnóstico suelen sugerirse ajustes técnicos que permitirían ampliar el uso de los dispositivos de almacenamiento, transmisión y comunicación de los conocimientos y la información producidos por la investigación. No es inusual que en respuesta se desate una ola de nuevas redes, seminarios con participación de académicos y formuladores de políticas, publicación de boletines y un sinnúmero de ingenios para facilitar el flujo de conocimientos desde los productores hasta los usuarios.

En cuanto a estos últimos, se sostiene que existiría la necesidad de pulir los sistemas de recepción y utilización del conocimiento, para lo cual se han ensayado diversas fórmulas como la constitución de equipos asesores a nivel ministerial, la "traducción" de resultados de investigación para uso de altos decisores, diversas formas de subcontratación de estudios guiados desde la demanda, etc.

Tras todas las medidas enunciadas permanece intacto el sueño, el anhelo, de que mediante ciertos ajustes técnicos en nuestros modos de producir, circular y usar conocimientos sería posible lograr una trayectoria relativamente simple y directa de convergencia entre esos conocimientos y la formulación de políticas, "toma de decisiones" y solución de problemas. Contra todos los buenos deseos, sin embargo, parece subsistir esa distancia que separa a los investigadores y los formuladores de políticas y el conocimiento que aquéllos generan no llega a ser utilizado por éstos. Pero, ¿existe realmente tal distancia y cuánto separa? 


\section{Una experiencia de la distancia que aparentemente separa la producción y la} utilización de conocimientos

Hace un tiempo me correspondió encabezar una Comisión a la cual el Gobierno le encomendó preparar un documento base para definir una política de desarrollo de la educación superior durante los años 90 y elaborar una propuesta de reforma de la legislación que regula a este sector. No pretendo aburrirlos con una crónica de esa experiencia sino extraer, directamente, algunas conclusiones que pudieran aprovecharse para el tema en discusión.

Primero que todo debo decir que los resultados finales de todo ese ejercicio, mirados a casi tres años de su ocurrencia, son más bien frustrantes. Digo bien: frustrantes; no frustrados completamente. La legislación que se buscaba cambiar no ha sido modificada, pero existe un proyecto de ley presentado al Parlamento. En cuanto al documento de políticas representa un aporte —más o menos valioso, según se lo quiera evaluar- y expresa algunas líneas de consenso en estas materias.

Creo que en la elaboración de ambas propuestas existió una utilización relativamente intensa de conocimientos acumulados, los cuales pudieron ser usados por constituir "conocimiento tácito", personal, corporizado, en cada uno de los miembros de esa Comisión. En cambio, cuando se necesité información y conocimientos específicos como por ejemplo, estudios sobre legislación internacional comparada en el campo de la educación superior, o proyecciones de matrícula hasta el año 2000, o información sobre rendimiento de las universidades y uso de sus recursos - habitualmente tal material no existía o no estaban disponibles. En algunos casos su ausencia podía suplirse mediante aportaciones realizadas contra demanda $o$ recurriendo a redes informales de especialistas.

Es interesante asimismo constatar que en varias ocasiones las necesidades más apremiantes tenían que ver con la posibilidad de contar con un tipo de conocimientos - 0 de estudios - que no son frecuentes en nuestras ciencias sociales. Me refiero a estudios y conocimientos que sí son de ocurrencia más o menos habitual en organismos como la CEPAL, el Banco Mundial o la OECD y que resultan del análisis de la actuación de sistemas o de la evaluación de políticas. Un ejemplo: el análisis comparado de diversas modalidades de financiamiento y asignación de recursos a las universidades, con una evaluación detallada de sus resultados en diversos países.

Una vez terminado el trabajo, la propuesta para reformar la legislación recibió el tratamiento habitual. Ingresó a una "arena de decisiones" y allí siguió, más o menos, la trayectoria que antes describí en términos más bien abstractos. O sea, quedó sometido a la interacción entre diversos agentes que inician el proceso de "arreglárselas" ("muddling through") para tratar de arribar a una decisión; en este caso, definir un proyecto de ley acorde con las necesidades y la visión del Poder Ejecutivo para ser presentado al Parlamento. Intervienen en esta etapa, por la naturaleza del asunto, diversas reparticiones de Gobierno en distintos momentos y niveles, más otra serie de actores que son atraídos a esta "arena": rectores más o menos influyentes de las universidades públicas, su organismo asociativo, rectores de universidades privadas, asociaciones de académicos, federaciones de estudiantes, sindicatos de trabajadores universitarios, representantes de partidos políticos, personeros de la Iglesia católica, altos jefes militares interesados en el destino de sus escuelas superiores, etc. 
A esa altura, lo que existe por tanto es un contexto interactivo donde operan múltiples racionalidades y donde las decisiones a que se espera arribar se hallan, en buena medida, indeterminadas, pues los procesos que conducen a ellas son autopropulsados por el juego de los agentes, cada uno dotado de sus propios intereses. Cada agente, a su vez, está premunido de conocimiento local y de información parcial, y actúa en un medio incierto a pesar de la presión que impone la modalidad burocrática a los procesos de toma de decisiones. El conocimiento provisto por la investigación juega ahora un rol cada vez más débil a medida que es más intenso el juego decisorio. En el mejor de los casos sirve propósitos tácticos: legitimar una decisión parcial, desplazar un argumento, ayudar a un giro posicional, contrarrestar una movida, etc.

\section{IV}

Con esto paso a la última parte; esta vez para dar una mirada más global sobre el contexto contemporáneo en que ocurren los procesos de utilización de conocimientos e investigación.

Por de pronto, cabe observar que los conocimientos tienen dos caras, una sola de las cuales aparece resaltada en las discusiones sobre su utilización e incidencia en las decisiones. En efecto, la cara más vista es la del conocimiento como representación, idea o bien simbólico; en cambio la otra cara, la que de común se halla oculta, es la del conocimiento como disposiciones y destrezas que permiten a su poseedor o actor un actuar informado, una práctica específica. El conocimiento-representación busca, antes que todo, comunicarse y obtener el reconocimiento de los demás productores al interior de las respectivas comunidades disciplinarias. En cambio, el conocimiento-destreza es practicado y su utilización se halla determinada por una estructura de oportunidades que está siempre más próxima al polo de la acción -y de las decisiones- que al polo de la producción. Desde cualquiera de ambos lados, el conocimiento es un material intangible que adquiere sentido interactivamente y se "realiza", por así decir, solamente a través de la interacción. Desde este punto de vista, nunca deja de ser utilizado.

Aquí, sin embargo, nos interesa sólo una clase de conocimiento -aquel producido por medio de la investigación social- y una sola dimensión de su utilización; aquella que tiene lugar en las diversas "arenas" donde se deciden asuntos que son objeto de la atención y el trabajo de los investigadores. Por su parte, estos últimos están viendo cambiar rápidamente su rol y su identidad profesional. Provenientes de una tradición que los emparentaba con la figura del intelectual, se acercaron después a la tradición del científico, incluso del técnico, mientras desarrollaban sus instrumentos de ingeniería política y social. Ahora estamos confrontados al hecho de que el conocimiento del que somos portadores está en alta demanda y se diversifica aceleradamente, al mismo tiempo que empieza a ser instrumentalizado por una variedad de agentes que difícilmente se acomodan a la descripción tradicional de un investigador social.

Para apurar la descripción de esos cambios traigo a colación a Robert Reich, profesor de la Kennedy School of Government de Harvard y actual Ministro de Trabajo de la administración Clinton. Según Reich, las intensas transformaciones que experimentan las sociedades a escala mundial como producto de la globalización de los mercados y la revolución tecnológica en curso, harían que desde ya, y cada vez con mayor nitidez en el futuro, se perfilen tres grandes categorías ocupacionales que denomina, respectivamente, servicios rutinarios de producción, servicios personales y servicios analítico-simbólicos. 
Aquí interesa solamente la última categoría, que comprende el conjunto de actividades que tienen que ver con la identificación, la solución y el arbitraje de problemas mediante la manipulación de conocimientos. Quienes están envueltos en tales actividades ganan su vida con la manipulación de símbolos: datos, palabras, representaciones orales y visuales; servicios que, como ocurre con diversos otros sectores de la economía, están sujetos al efecto de una rápida globalización de sus mercados. Según señala Reich, esta categoría ocupacional incluye a un heterogéneo grupo de personas que usualmente se llaman a sí mismos científicos; ingenieros de diseño, de software, de biotecnología y de sonido; ejecutivos de relaciones públicas; abogados (pero no todos, sino dependiendo del tipo de trabajo que desempeñan); banqueros de inversión; ejecutivos de desarrollo inmobiliario; e incluso algunos contadores de alto vuelo. También se incluye en esta categoría buena parte del trabajo realizado por consultores de management, financieros y tributarios; consultores de arquitectura; especialistas en información para la administración, en desarrollo organizacional y de recursos humanos; planificadores estratégicos, "cazadores de cabezas" y analistas de sistemas. También: ejecutivos de publicidad, estrategas de marketing, directores de arte, cineastas, escritores y editores, periodistas, músicos, productores de televisión y cine.

Tres rasgos parecen ser característicos del tipo de trabajo que desarrollan los analistas simbólicos:

- identifican, solucionan o arbitran problemas mediante la manipulación de símbolos para lo cual emplean instrumentos analíticos aguzados por la experiencia;

- habitualmente sus ingresos no están ligados al tiempo que emplean en producir sus servicios sino a la calidad, originalidad, oportunidad e inteligencia de los mismos y, ocasionalmente, a la rapidez con que identifican, resuelven o arbitran problemas; y, tercero,

- sus carreras profesionales no son lineales o jerárquicas sino que proceden a lo largo de una trayectoria que depende en gran medida de su capacidad de trabajo, prestigio acumulado, participación en redes o inclusión en equipos, etc.

Podría sostenerse que los profesionales a quienes tradicionalmente hemos llamado investigadores sociales, incluidos los investigadores educacionales, forman parte - por lo menos un sector de ellos- de esta emergente categoría de analistas simbólicos. Su antiguo rol -la producción de conocimientos para ser usados por terceros -está cambiando rápidamente. En efecto, hoy se espera, y el mercado demanda, a personas en disposición de producir, transportar, usar y aplicar conocimientos para la identificación, resolución y arbitraje de problemas; en general, personas con la habilidad de manipular conocimientos para el cumplimiento de esas funciones, sea por sí mismas o mediante la identificación de otros analistas, equipos o redes que pueden disponer de esos conocimientos.

Hay dos otras maneras posibles de representarse esos cambios: primero, todo ocurre como si la distancia entre la producción de conocimientos -dominio reservado antiguamente al investigador - y su utilización se estuviese comprimiendo hasta fusionarse en muchos puntos; segundo, todo ocurre como si el lado práctico del conocimiento - es decir, esa parte del mismo investida en las destrezas y capacidades del individuo entrenado para manipular conocimientos- estuviese llegando a significar cada vez más en contraste con el tradicional predominio de la cara idealista o de contenido meramente representacional del conocimiento. 
Los investigadores sociales recluidos en sus dominios tradicionales de producción trátese de departamentos universitarios o centros académicos de investigación- se encuentran cada día en mayor desventaja respecto a los analistas simbólicos que cumplen similares funciones en los nuevos dominios, como pueden ser ciertos "think tanks", oficinas consultoras privadas, grupos de asesoría legislativa, agencias de análisis de diverso tipo, ciertos organismos internacionales y, en general, redes de analistas simbólicos cuyos miembros se conectan sueltamente con la estructura de oportunidades que ofrece el mercado en expansión para los servicios de manipulación de conocimientos.

En efecto, el viejo esquema triangular de producción, difusión y utilización da paso ahora a un sistema que se asemeja cada vez más a un contexto de mercado dentro del cual se organizan los servicios desarrollados por los analistas simbólicos. Dicho mercado, si puede usarse este lenguaje, valoriza el servicio final más que el conocimiento-ideal involucrado en las complejas y sutiles actividades de identificación, solución y arbitraje de problemas. Supone el empleo de conocimientos, en la cantidad y de la calidad que sean necesarios, pero no valoriza directamente al conocimiento como un bien simbólico, sino el servicio que lo manipula y opera los efectos prácticos buscados.

En suma, la investigación como tal —o sea, como operación metódica destinada a descubrir conocimientos y a ponerlos en circulación para que estando en órbita otros agentes utilizadores los empleen y apliquen a las decisiones que están a la mano - experimenta en la actualidad una verdadera mutación. Pasa a integrarse, como un componente más, dentro de una noción de servicio que, sin embargo, la desborda por todos lados, especialmente en dirección de lo que podemos llamar "practicas de análisis simbólico aplicado".

Algunos investigadores sociales perciben este movimiento como una amenaza. En efecto, su actividad tradicional empieza a encontrar dificultades para ser financiada y sus productos - conocimientos impresos, de común- parecen no ingresar a ningún circuito efectivo de utilización, mucho menos a las "arenas de decisión" de asuntos relevantes. De otra parte, se sostiene que bajo la presión de los reacomodos que experimenta el mercado del análisis simbólico, el tiempo requerido para investigar seria $y$ productivamente se reduce, al punto que la investigación empieza a concebirse como acción contra demanda, de corto plazo, de escaso contenido teórico o conceptual y sujeta a una agenda de problemas que no serían necesariamente los más significativos desde el punto de vista interno del campo de investigación. Como efecto de todo esto se teme, además, que la investigación social pudiera perder uno de sus rasgos más apreciados por los propios investigadores: su carácter crítico.

La profunda reestructuración que está experimentando el dominio del análisis simbólico permite poner en duda algunas de esas aprehensiones, formuladas bajo la inspiración de los viejos parámetros de organización del campo de la investigación social.

En efecto, si algo cabe observar es que el financiamiento para este tipo de actividades en vez de disminuir se ha ensanchado, sólo que bajo nuevas modalidades las cuales tornan obsoletas aquellas que en el pasado permitieron el desarrollo de las universidades y los centros académicos de investigación. Así, por ejemplo, la globalización del mercado de los analistas simbólicos redefine las viejas relaciones de cooperación internacional, sustituyendo las modalidades de financiamiento benevolente o solidario por modalidades ahora condicionadas desde el lado de la demanda. En seguida, la tradicional vía de asignación automática de recursos públicos para la investigación social —que beneficiaba casi exclusivamente a las universidades -alcanza un punto de 
relativo estancamiento, pero a su lado se multiplican las demandas públicas y privadas de servicios prestados por los analistas sociales en nuevos campos ligados al desarrollo organizacional, la planificación estratégica, el diseño de sistemas, la formación y reorientación de recursos humanos, el marketing y la publicidad, la subcontratación de funciones públicas, la evaluación de productos y conocimientos, etc.

De manera semejante, al mismo momento en que parece evidenciarse un quiebre o agotamiento del modelo tradicional de utilización del conocimiento producido por la investigación social, surge en paralelo o sustitutivamente un nuevo contexto que demanda más y más conocimientos bajo la forma de consultorías, asesorías y un sinnúmero de servicios de identificación, solución y arbitraje de problemas en múltiples "arenas de decisión". Por todas partes surgen nuevos roles del estilo de los llamados "creativos", que hace rato desbordaron el campo de la publicidad. Asimismo, se multiplican las funciones de diseño de sistemas sociales, de consejería comunicacional, de desarrollo y evaluación de procesos, de administración y ejecución de estudios estratégicos, de aplicación de conocimientos organizacionales, todos los cuales dan lugar a una rica y densa actividad de generación y uso de conocimientos.

Bajo las nuevas condiciones el tiempo de maduración de las prácticas "clásicas" (o académicas) de investigación tiende efectivamente a desaparecer, mientras aumenta la velocidad de los tráficos del conocimiento y la información, sea el momento de su gestación, circulación o incorporación a una variedad de "arenas de decisión" y esferas de utilización. Parece entonces como si la investigación social siguiera operando al ritmo dictado por la máquina a vapor, mientras a su alrededor los conocimientos y la información se desplazan a la velocidad de las señales electrónicas. En realidad, nada indica que el volumen de la producción científica pudiera estar amenazado. Todo lo contrario. Según muestran las estadísticas, la publicación de revistas científicas ha venido multiplicándose por dos cada 15 años desde el siglo XVII, habiendo aumentado de 10.000 alrededor a comienzos del presente siglo a 100.000 en 1990 (Rosovsky, 1990: 102). Más impresionante es lo que ha ocurrido en el ámbito de la información. El costo real de almacenar, procesar y transmitir una unidad de información ha estado cayendo en un $20 \%$ anual durante los últimos 40 años (Banco Mundial, 1992). Luego, resulta del todo previsible que el tiempo de la investigación social tenga que adaptarse a las cambiantes condiciones de la producción y circulación de conocimientos e información. Muchas veces, más importante que generar lo que suele llamarse "nuevos conocimientos" —que en nuestros dominios frecuentemente no son tales sino variaciones sobre el conocimiento dado-, es tener la capacidad, en el país, para aprovechar efectivamente los conocimientos disponibles. Por su lado, esa labor de "aprovechamiento" suele ser tan compleja y apasionante, y conducir por caminos tan imprevisibles, que en no pocas ocasiones da lugar a verdaderos "descubrimientos", como resulta ser, por ejemplo, la "traducción" y "aplicación" de las teorías heideggerianas y de ciertas escuelas lingüísticas al dominio del diseño organizacional.

Tampoco resulta claro, como temen algunos investigadores, que en los nuevos contextos de manipulación de conocimientos se deba producir, necesariamente, una desvalorización -incluso, muerte- de la teoría en favor de un tratamiento meramente pragmático de los conocimientos. Ocurre aquí algo semejante a lo que ha venido sucediendo en las ciencias naturales. Como se sabe, allí la distinción entre investigación y desarrollo es cada vez más difícil de percibir y el tiempo entre un descubrimiento y su aplicación ha tendido a reducirse vertiginosamente. Lo mismo parece estar ocurriendo en el campo del análisis social. La tajante separación entre producción de conocimientos como representaciones ideales — pensada para producir información o comprensión que 
vaya más allá del conocimiento o de la práctica aceptada (Ziman, 1986: 148) — y su puesta en acto mediante la incorporación de ideas nuevas y nuevas prácticas en los procesos sociales y políticos tiende a debilitarse y se transforma en un continuo. Muchos trabajos teóricamente sugerentes surgen de prácticas que poco tienen que ver con la actividad tradicional de investigación académica y ésta, cada vez que sus practicantes se ven envueltos en actividades que en el mundo de las ciencias naturales se llamarían un desarrollo o tecnológicas, suele por ese solo contacto adquirir una mayor velocidad, productividad e interés.

¿Es posible que la investigación social entendida como actividad de analistas simbólicos en un mercado de servicios retenga la dosis de capacidad crítica que su tradición reclama como uno de sus mayores logros? En verdad, la cuestión esencial ha sido siempre cómo explicar el mundo social en orden a transformarlo, y no cómo obtener satisfacción o sacar provecho del acto de su negación informada. Si tal es el objeto, no veo cómo podría temerse que una actividad íntimamente comprometida con la transformación del mundo social a través de la manipulación de conocimientos podría perder sus aristas críticas. Más bien, la crítica -y allí reside la dificultad o desafío- está llamada ahora a encarnarse en esas prácticas propias del analista simbólico, sin poder recurrir a la cortada que significa decir que los conocimientos producidos no son utilizados por haber sido pensados con una orientación o bajo supuestos paradigmáticos que son incompatibles con aquéllos que comparten los decisores o la gente allá afuera, en el mundo que se desea afectar mediante la investigación.

En suma, parece haber llegado el momento en que el conocimiento deja de ser el dominio exclusivo de los intelectuales y sus herederos más especializados investigadores y tecnócratas- para convertirse en un medio común a través del cual las sociedades se organizan, cambian y adaptan. De aquí en adelante corresponde a los investigadores sociales ajustarnos a esa nueva situación o Corremos el riesgo de convertirnos en una comunidad marginal.

\section{BIBLIOGRAFIA}

Banco Mundial (1992), World Bank Policy Research Bulletin, vol. 3, No. 2.

BRIONES, Guillermo (1990), Generación, diseminación y utilización del conocimiento en Educación; FLACSO, Santiago de Chile (3 volúmenes).

CORBALAN, Ana María (1987), "Estado acerca del uso de la información en la toma de decisiones en educación en América Latina", BRIDGES, Santiago de Chile.

JOHNSON, Paul (1988), Intellectuals; Weidenfeld and Nicholson, London.

LINDBLOM, Charles (1990), Inquiry and Change; Yale University Press and Russell Sage

LINDBLOM, Charles y COHEN, David (1979), Usable Knowledge, Yale University Press.

KOLAKOWSKI, Leszek (1990), La modernidad siempre a prueba; Vuelta, México. 
LINDBLON, Charles (1990), Inquiry and Change; Yale Uníversity Press and Russell Sage Foundation, New York.

LINDBLON, Charles y COHEN, David (1979), Usable Knowledge, Yale University Press.

REICH, Robert (1992), The Work of Nations; Vintage Books, New York.

ROSO VSKY, Henry (1990), The University. An Owner's Manual; W.W. Norton \& Company, New York.

WEISS, Carol (1979), "The many meaníngs of research utilization"; Public Administration Review, Vol. 39, No. 5.

ZIMAN, John (1986), Introducción al Estudio de las Ciencias, Editorial Ariel, Barcelona. 